\title{
$\mathbb{\Delta}$ Economics Bulletin
}

\author{
Volume 33, Issue 2
}

\section{Willingness to pay for intervention policies related to HIV/AIDS: a theoretical framework with endogenous risk, perceived effectiveness and altruism}

Mario Andres Fernandez

Texas A\&M University
Douglas Shaw

Texas A\&M University

\begin{abstract}
Intervention policies for HIV/AIDS differ in their approaches, and acceptability to control the spread of the epidemics. We introduce a theoretical model to depict how several factors may determine an individual's maximum willingness to pay for any HIV/AIDS policy. Heterogeneous baseline risks, through risky sexual activities, operate so that those individuals with higher exposure to risky activities are inclined to pay for any policy that reduces the risk. However, given the epidemiological and economic context of a geographic region, the perceived effectiveness of a policy implies that it will reach some level of risk reduction outcome and then, not contribute any further to the control of epidemics. What comes into play is whether individuals observe the risk change generated by the policy and implications for behavioral changes. Finally, we also introduce altruistic motives as a determinant of the WTP.
\end{abstract}

\footnotetext{
Acknowledgements The authors thank James Hammitt, Paul Jakus, Jason Shogren, Paan Jindapon and Kerry Smith for helpful comments on a much earlier version of the manuscript. Conflicts of Interest Authors do not have any conflict of interest

Citation: Mario Andres Fernandez and Douglas Shaw, (2013) "Willingness to pay for intervention policies related to HIV/AIDS: a theoretical framework with endogenous risk, perceived effectiveness and altruism", Economics Bulletin, Vol. 33 No. 2 pp. 1457-1467.

Contact: Mario Andres Fernandez - fernandezeas@gmail.com, Douglas Shaw - wdshaw@tamu.edu.

Submitted: February 15, 2013. Published: June 14, 2013.
} 


\section{INTRODUCTION}

Disease epidemics such as acquired immunodeficiency syndrome (AIDS) affect millions of people and present challenges to policy-makers for the design and implementation of policies to stop the disease's spread, or to reduce adverse impacts once it is contracted. According to the World Health Organization (www.who.int) 35 million people have died since the beginning of the AIDS epidemic, and while fewer people are dying today as compared to a decade ago, 1.7 million died in 2011 alone. Testing and treatment have been effective. Testing may be reducing the spread of infection, as HIV incidence has fallen across 33 countries, and 22 of these are in sub-Saharan Africa, the region most affected by the AIDS epidemic. Nevertheless, in 2010, there were between 2.4 million to2.9 million new HIV infections, which was down $21 \%$ from the peak of the global epidemic in 1997; 95 million people world-wide were tested, and about half of pregnant women who were HIV positive received medical treatments to prevent transmission from mother to child (UNAIDS, 2012). From 2010 to 2012 there was a $60 \%$ increase in the number of people receiving treatment that saves their lives: 8 million were on antiretroviral treatment $(A R T)$.

HIV/AIDS policies involve different levels of resource allocation where outcomes are conditional on factors such as (i) individual perception of infection risk; (ii) the heterogeneity of baseline risks, which depends on sexual behavior and the use of preventative measures; (iii) the nature and size of proposed risk changes because of health policies, and (iv) vulnerability stemming from poverty levels, age, and gender gaps, (e.g. Bignami-van Assche et al., 2007, Hammit and Treich 2007). Altruism may also affect policies because it is likely that some individuals might be willing to pay to support, protect or comfort others against an HIV/AIDS infection (Ainsworth et al., 2005). With thinning budgets tied to slow economic growth or recession, policy makers may be forced to make difficult choices. That is, policies that result in large risk reductions to a high-risk population could be socially preferable to those that provide smaller risk reductions to a wider proportion of the population (Prinja 2011). Some have argued that ART may demand higher budget levels and be at least as cost effective in reducing HIV infection risk as any of the other type interventions, but all of this is still debatable (Johnston et al. 2010; McCabe 2010). Thus, how to focus spending is of increasing importance, and this is tied to close scrutiny of preferences both for those potentially infected, and for those who might support interventions for other reasons.

Underlying the economics of efficient intervention policy design is the concept that marginal damage from the number of HIV/AIDS-infected people should equal the marginal cost of reducing the number. To reach this efficient level one might envision a subsidy policy of some type, and in order to inform its level one needs information on average maximum willingness to pay (WTP). Then, the contribution of this paper is to provide a model of individual WTP for some type of policy that reduces the HIV-infection risk, where optimal resource allocation conditions are derived, and implications of the model are considered. The WTP approach offers advantages over other forms of economic evaluation because no limitation is placed on what factors are included in the evaluative space (Shiell and Rush 2003, Pratt and Zeckhauser 1996). The remainder of this paper is organized as follows. Section two presents the WTP model. Section three offers some conclusions. 


\section{THE MODEL}

One aspect of the WTP approach is its capacity to capture a broader range of utilitybearing effects from health-care services or any health-related policy (Shiell and Rush 2003). The formal derivation of an individual-specific WTP essentially determines the change in income that is equivalent to the welfare related to the provision of any policy. Because individuals are not identical, information and heterogeneity may have a large effect on an individual expression for WTP. A typical assumption is that maximum WTP to reduce health risks increases more rapidly for an individual whose risk is increased than it decreases for an individual facing a risk reduction: that is, the indifference curve for wealth and risk is convex (Hammitt and Treich 2007).

An individual values a risk reduction by considering expected utility, maximizing this subject to budget constraints. The $k^{\text {th }}$-individual's problem is to maximize state-dependent expected utility:

$\varphi_{k}=p_{k}\left(e_{k}, x, \bar{p}_{k}\right) u_{k}\left(w_{k}\right)+\left(1-p_{k}\left(e_{k}, x, \bar{p}_{k}\right)\right) v_{k}\left(w_{k}\right)$

where $p_{k}$ is the endogenous probability of staying healthy (not getting infected), $e_{k}$ is referred as self protection (i.e. condom use), $x$ the proposed risk change, $\bar{p}_{k}$ is an exogenous health risk. The latter could be a science-based probability of not getting infected, perhaps common to individuals belonging to vulnerable population groups. Note that in equation (1) $w_{k}$ is wealth, $u_{k}\left(w_{k}\right)$ and $v\left(w_{k}\right)$ are the utility levels of wealth if not HIV-infected, and if HIV-infected, respectively. ${ }^{1}$ Because the model refers to attitudes and self-protection related to sexual behavior, abstinence behavior is not considered here.

We conventionally assume that $u$ and $v$ are twice differentiable, $u_{k}>v_{k}, u_{k}^{\prime}>v_{k}^{\prime}$, $u_{k}^{\prime \prime} \leq 0$, and $v_{k}^{\prime \prime} \leq 0$. That is, at any wealth level both utility and marginal utility are larger if non-infected than if infected (Hammitt and Treich, 2007). The value of a risk reduction (we use the abbreviation VRR below) is determined by the marginal rate of substitution between $w_{k}$ and $x$, and is defined by

$V R R_{k}=-\frac{d w_{k}}{d x}=\frac{\left(u_{k}\left(w_{k}\right)-v_{k}\left(w_{k}\right)\right) \frac{\partial p_{k}\left(e_{k}, x, \bar{p}_{k}\right)}{\partial x}}{p_{k}\left(e_{k}, x, \bar{p}_{k}\right) u_{k}^{\prime}\left(w_{k}\right)+\left(1-p_{k}\left(e_{k}, x, \bar{p}_{k}\right)\right) v_{k}^{\prime}\left(w_{k}\right)}$

This expression serves as the starting point to study how information about individual baseline risks affects the valuation of changes in risk or probability (Jones-Lee, 1992).

\subsection{Heterogeneity in baseline risk}

Let $\theta_{k}(x)$ be the WTP for a risk-reducing policy such that:

$$
\begin{array}{r}
p_{k}\left(e_{k}, x, \bar{p}_{k}\right) u_{k}\left(w_{k}-e_{k}-\theta_{k}(x)\right)+\left(1-p_{k}\left(e_{k}, x, \bar{p}_{k}\right)\right) v_{k}\left(w_{k}-e_{k}-\theta_{k}(x)\right) \\
=p_{k}\left(e_{k}, 0, \bar{p}_{k}\right) u_{k}\left(w_{k}-e_{k}\right)+\left(1-p_{k}\left(e_{k}, 0, \bar{p}_{k}\right)\right) v_{k}\left(w_{k}-e_{k}\right)
\end{array}
$$

\footnotetext{
${ }^{1}$ A reviewer notes that we have not explicitly introduced the role of insurance here, which would of course influence WTP, so our approach here is perhaps not well suited to a setting where all HIV infected patients are guaranteed adequate health insurance or coverage.
} 
where $x$ is the risk reduction, assumed as a public-good in this section, and $0 \leq x<p_{k}$. This is the usual expression that relates to an option price welfare measure (Graham, 1981). Differentiating (3) with respect to $x$,

$$
\theta_{k}^{\prime}(x)=\frac{p_{k}^{\prime}\left(u_{k}\left(w_{k}-e_{k}-\theta_{k}(x)\right)-v_{k}\left(w_{k}-e_{k}-\theta_{k}(x)\right)\right)}{p_{k} u_{k}^{\prime}\left(w_{k}-e_{k}-\theta_{k}(x)\right)+\left(1-p_{k}\right) v_{k}^{\prime}\left(w_{k}-e_{k}-\theta_{k}(x)\right)}
$$

Then, in order for $\theta_{k}^{\prime}(x)>0$ to hold, $p_{k}^{\prime}=\frac{\partial p_{k}\left(e_{k}, x, \bar{p}_{k}\right)}{\partial x}>0$. That is, any individual observes, or at least correctly perceives, the existence of a significant utility difference between both states of nature, and identifies a positive relation between the risk change generated by the policy and the individual baseline risk. We focus on the convexity of the WTP function, because this reveals how information on heterogeneous baseline risks affects the WTP. Differentiating (4) with respect to $x$ :

$$
\theta_{k}^{\prime \prime}(x)=\frac{p_{k}^{\prime \prime}\left(u_{k}-v_{k}\right)-\theta_{k}^{\prime} p_{k}^{\prime}\left(u_{k}^{\prime}-v_{k}^{\prime}\right)}{\left(p_{k} u_{k}^{\prime}+\left(1-p_{k}\right) v_{k}^{\prime}\right)^{2}}\left(p_{k}^{\prime}\left(u_{k}^{\prime}-v_{k}^{\prime}\right)-\theta_{k}^{\prime}\left(p_{k} u_{k}^{\prime \prime}+\left(1-p_{k}\right) v_{k}^{\prime \prime}\right)\right)
$$

Where $u_{k}(\cdot)=u_{k}\left(w_{k}-e_{k}-\theta_{k}(x)\right)$ and $v_{k}(\cdot)=v_{k}\left(w_{k}-e_{k}-\theta_{k}(x)\right)$. The second term in the right-hand side of (5) is positive, and in order for the WTP function to be concave $\left(\theta_{k}^{\prime \prime}(x)<0\right)$, then $p_{k}^{\prime \prime}=\frac{\partial^{2} p_{k}\left(e_{k}, x, \bar{p}_{k}\right)}{\partial x^{2}}<0$ must hold. In other words, those with relatively low values of $p_{k}$, that is, with active sexual behavior and low values of $e_{k}$, will have a higher marginal WTP for increases in $x$, as compared to those with higher $p_{k}$. However, risk changes generated by policies have marginally decreasing effects on the probability of remaining healthy.

\subsection{Policy effectiveness}

Suppose a planner must allocate a fixed budget among policies (Corso et al., 2002). This budget greatly changes when one contemplates subsidized or "free" assured public health care or insurance, but do not explicitly consider that here. Let a representative agent of society's risk reduction $x\left(\epsilon_{i}\right)$, be a non-decreasing function of a parameter $\epsilon_{i}\left(\frac{d x}{d \epsilon_{i}}=x_{\epsilon_{i}}^{\prime} \geq\right.$ $0)$. The term $\epsilon_{i}$ may be observed as an index of the perceived effectiveness of the $i^{\text {th }}$-policy so that it is possible to set an ordering. Here we incorporate an individual's perception regarding any policy's effectiveness which determine WTP and redefine non-infection probability as $p_{k i}=p_{k i} e_{k}, x, \theta_{k}\left(x\left(\epsilon_{i}\right)\right)$.

Suppose for any two different policies, $(i, j)$, an individual perceives $x\left(\epsilon_{i}\right) \geq x\left(\epsilon_{j}\right)$, such that $p_{k i} \geq p_{k j}, \theta_{k}\left(x\left(\epsilon_{i}\right)\right) \geq \theta_{k}\left(x_{k}\left(\epsilon_{j}\right)\right)$ and

$$
\begin{aligned}
p_{k i} u_{k}\left(w_{k}-e_{k}-\theta_{k}\left(x\left(\epsilon_{i}\right)\right)\right)+\left(1-p_{k i}\right) v_{k}\left(w_{k}-e_{k}-\theta_{k}\left(x\left(\epsilon_{i}\right)\right)\right) \\
=p_{k i}\left(e, 0, \bar{p}_{k}\right) u_{k}\left(w_{k}-e_{k}\right)+\left(1-p_{k i}\left(e, 0, \bar{p}_{k}\right)\right) v_{k}\left(w_{k}-e_{k}\right)
\end{aligned}
$$


For $f \in(i, j)$, let $\frac{d \theta\left(x\left(\epsilon_{f}\right)\right)}{d \epsilon_{f}}=\theta_{k, \epsilon_{f}}^{\prime}, u_{k f}(\cdot)=u_{k f}\left(w_{k}-e_{k}-\theta_{k}\left(x\left(\epsilon_{f}\right)\right)\right), \quad v_{k f}(\cdot)=$ $v_{k}\left(w_{k}-e_{k}-\theta_{k}\left(x\left(\epsilon_{f}\right)\right)\right), u_{k f}^{\prime}(\cdot)=\frac{d u_{k f}}{d \theta_{k}}, \quad v_{k f}^{\prime}(\cdot)=\frac{d v_{k f}}{d \theta_{k}}, u_{k f}^{\prime \prime}(\cdot)=\frac{d^{2} u_{k f}}{d \theta_{k}^{2}}$, and $\quad v_{k f}^{\prime \prime}(\cdot)=$ $\frac{d^{2} v_{k f}}{d \theta_{k}^{2}}$

Differentiate (6) with respect to $\epsilon_{i}$ :

$$
\theta_{k, \epsilon_{i}}^{\prime}=\frac{p_{k i}^{\prime}\left(u_{k i}(\cdot)-v_{k i}(\cdot)\right)}{p_{k i} u_{k i}^{\prime}(\cdot)+\left(1-p_{k i}\right) v_{k i}^{\prime}(\cdot)}>0
$$

For $\theta_{k, \epsilon_{i}}^{\prime}>0$, it is simultaneously required that $\frac{d p_{k}\left(e_{k}, x, \theta_{k}\left(x\left(\epsilon_{i}\right)\right)\right)}{d x}=p_{k i}^{\prime}>0$, and $\frac{d x}{d \epsilon_{i}}=x_{\epsilon_{i}}^{\prime} \geq 0$, which imply positive implications over the achievement of $x\left(\epsilon_{i}\right)$, that is, individuals should perceive the policy as acceptable $\left(p_{k i}^{\prime}>0\right)$ and engage in sexual behavior and self-defense expenditure such that the difference $u_{k i}(\cdot)-v_{k i}(\cdot)$ remains significant. Differentiating (7) with respect to perceived effectiveness, $\epsilon_{i}$, yields

$$
\begin{aligned}
\theta_{k, \epsilon_{i}}^{\prime \prime}= & \frac{\left(x_{\epsilon_{i}}^{\prime}\right)^{2}\left(u_{k i}(\cdot)-v_{k i}(\cdot)\right)\left(p_{k i}^{\prime \prime}-p_{k i}^{\prime} \theta_{k i}^{\prime}\right)}{\left(p_{k i} u_{k i}^{\prime}(\cdot)+\left(1-p_{k}\right) v_{k i}^{\prime}(\cdot)\right)^{2}} . \\
& \cdot\left(p_{k i}^{\prime}\left(u_{k i}^{\prime}(\cdot)-v_{k i}^{\prime}(\cdot)\right)-\theta_{k, \epsilon_{i}}^{\prime}\left(p_{k i} u_{k i}^{\prime \prime}(\cdot)+\left(1-p_{k}\right) v_{k i}^{\prime \prime}(\cdot)\right)\right)
\end{aligned}
$$

The numerator of the first term of the right hand side of (8) is negative, whereas the second term is positive, then $\theta_{k, \epsilon_{i}}^{\prime \prime}<0$. Thus, the WTP function is concave with respect to $\epsilon_{i}$. That is, as the intervention generates a larger risk reduction, it will eventually reach a maximum, observable for the agent, where it should be supplemented or replaced by another policy, as determined by the spread of the epidemic. From (7) and (8), a positive perception of the $i^{t h}$-policy's effectiveness, that is, a larger $x\left(\epsilon_{i}\right)$, motivates both a larger marginal WTP for its implementation and an increase of the concavity of the WTP function.

Now from (7), for $f \in(i, j)$, let $\theta_{k, \epsilon_{f}}^{\prime}=\frac{p_{k f}^{\prime}\left(u_{k f}(\cdot)-v_{k f}(\cdot)\right)}{p_{k f} u_{k f}^{\prime}(\cdot)+\left(1-p_{k f}\right) v_{k f}^{\prime}(\cdot)}$, such that

$$
\frac{\theta_{k, \epsilon_{i}}^{\prime}}{\theta_{k, \epsilon_{j}}^{\prime}}=\frac{\frac{p_{k i}^{\prime}\left(u_{k i}(\cdot)-v_{k i}(\cdot)\right)}{p_{k i} u_{k i}^{\prime}(\cdot)+\left(1-p_{k i}\right) v_{k i}^{\prime}(\cdot)}}{\frac{p_{k j}^{\prime}\left(u_{k j}(\cdot)-v_{k j}(\cdot)\right)}{p_{k j} u_{k j}^{\prime}(\cdot)+\left(1-p_{k j}\right) v_{k j}^{\prime}(\cdot)}}
$$

Equation (9) shows that for the marginal WTP for the $i^{t h}$ policy to be greater than the one for $j^{t h}$, then the effect of $x_{k}$ over the probability of remaining healthy (non-infected) should be greater on $i$. Plus, the utility differences under both policies should remain significant and larger for $i$ with respect to $j$. Comparing between infection and no-infection 
states, the trade-off between policies can be observed in terms of $\epsilon_{f}$, the risk change $p_{k}^{\prime}$ and the individual's actions for the utility difference to remain significant.

Suppose $\epsilon$ can be mapped over a continuum and the individual is capable of setting an ordering of different policies in terms of their perceived effectiveness. Figure 1 shows a summary of how risk changes and perceived effectiveness affect individual WTP. First, a credible risk change, $x$, motivates an increase in WTP which is accompanied by an increase in the perceived effectiveness. Conversely, if for a proposed policy, the perceived effectiveness increases, which also motivate an increase in WTP, then the policy's expansion would allow an increase of $x$ on the probability of remaining healthy.

The main implication from (7) and (8) is that a particular policy will not contribute to further changes in the probability of remaining non-infected, reaching a maximum at $p_{\max }^{\prime}$ and $\epsilon_{\max }^{\prime}$ (Prohaska 1990). In practice, in a country where the prevalence level of HIV/AIDS is less than 1\%, it is recommended IEC policies (Jamison 2006). Yet, when the epidemic grows reaching prevalence level higher than 5\%, IEC policies should be supplemented with $A R T$ or condom promotion (ibid). This situation is reflected in WTP' which depicts that the probability of remaining healthy cannot surpass $p_{\max }^{\prime}$, and the individual will not show a positive marginal $W T P$ for this particular policy. Once a new policy is to be implemented, this motivates a shift to $W T P^{\prime \prime}$ provided it is perceived as effective and motivates a significant risk change.

No matter how effective a policy is perceived and how it is implemented, we allow for the possibility that $p_{k}$ does not reach 1 since there are issues on individual behavior that cannot be controlled by public health policies.

Figure 1: Willingness to Pay, Baseline Risk and Perceived Policy Effectiveness

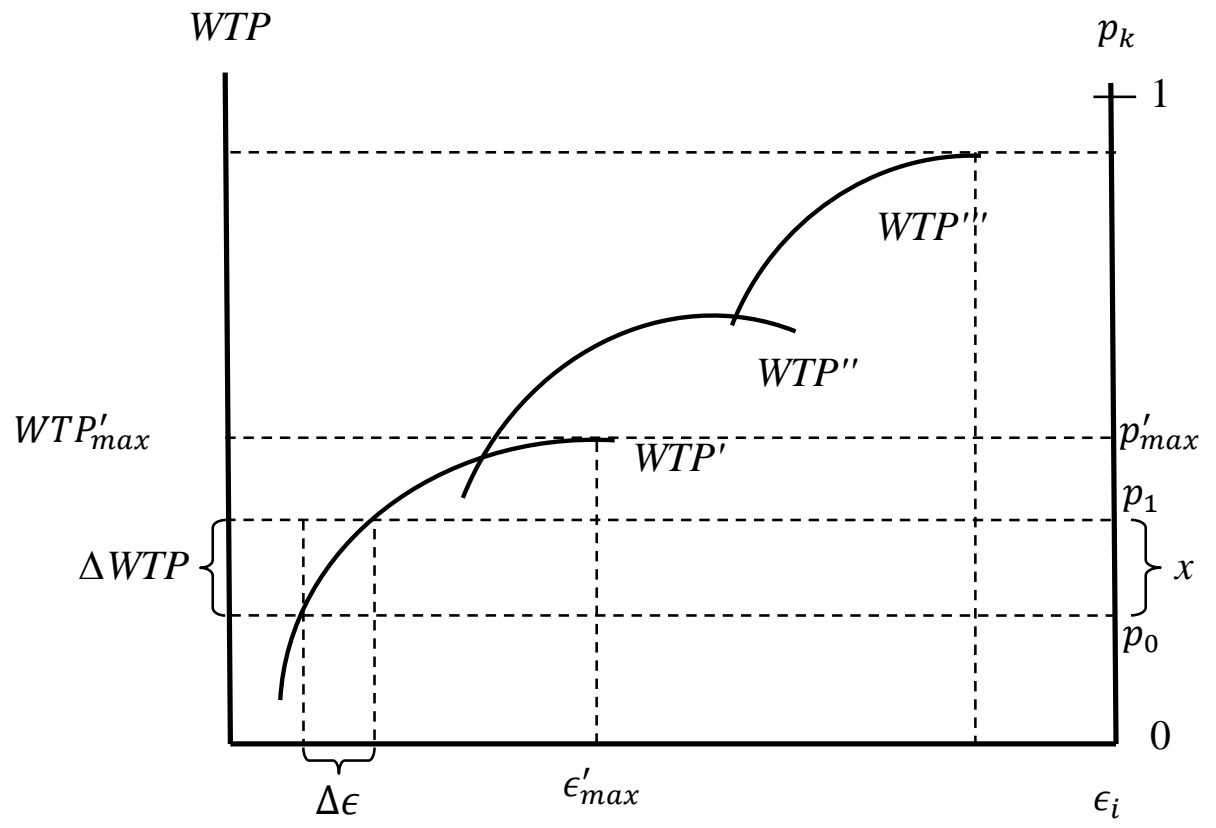




\subsection{Altruism}

Because of the implications HIV/AIDS has for all of society, altruistic values are potentially entangled in any individual WTP (Araña and León, 2002). WTP might well capture not only an individual's motives for self-protection, but in fact all components of value (Shiell and Rush, 2003). Thus, it becomes potentially important to investigate changes in WTP related to improvements in the health and safety of others in addition to one's selfprotection (Hurley and Mentzaki, 2011). Suppose there are two individuals, where $\theta_{m}\left(x_{m}, x_{n}\right)$ is the WTP of individual $m$, and is a function of his risk change $\left(x_{m}\right)$ but also of $n\left(x_{n}\right)$. Let $x_{m} \neq x_{n}$, that is, the risk changes pertain to separate population groups, or each risk changes represents different sexual behaviors. In this case the nature and impact of HIV/AIDS policies differ. From a social perspective we have:

$$
\begin{aligned}
p_{m}\left(e_{m}, x_{m}, \bar{p}_{m}\right) & u_{m}\left(w_{m}-e_{m}-\theta_{m}\left(x_{m}, x_{n}\right)\right) \\
+ & \left(1-p_{m}\left(e_{m}, x_{m}, \bar{p}_{m}\right)\right) v_{m}\left(w_{m}-e_{m}-\theta_{m}\left(x_{m}, x_{n}\right)\right)+ \\
& \quad+p_{n}\left(e_{n}, x_{n}, \bar{p}_{n}\right) u_{n}\left(w_{n}-e_{n}-\theta_{n}\left(x_{m}, x_{n}\right)\right) \\
+ & \left(1-p_{n}\left(e_{n}, x_{n}, \bar{p}_{n}\right)\right) v_{n}\left(w_{n}-e_{n}-\theta_{n}\left(x_{m}, x_{n}\right)\right) \\
& =p_{m}\left(e_{m}, 0, \bar{p}_{m}\right) u_{m}\left(w_{m}-e_{m}\right)+\left(1-p_{m}\left(e_{m}, 0, \bar{p}_{m}\right)\right) v_{m}\left(w_{m}-e_{m}\right) \\
+ & p_{n}\left(e_{n}, 0, \bar{p}_{n}\right) u_{n}\left(w_{n}-e_{n}\right)+\left(1-p_{n}\left(e_{n}, 0, \bar{p}_{n}\right)\right) v_{n}\left(w_{n}-e_{n}\right)
\end{aligned}
$$

Set $\quad p_{k, l}^{\prime}=\frac{d p_{k}\left(e_{k}, x_{k}, \bar{p}_{k}\right)}{d x_{k}}, \quad \theta_{k, l}^{\prime}\left(x_{m}, x_{n}\right)=\frac{d \theta_{k}\left(x_{m}, x_{n}\right)}{d x_{l}}, \quad u_{k}(\cdot)=u_{k}\left(w_{k}-e_{k}-\theta_{k}\left(x_{m}, x_{n}\right)\right)$, $v_{k}(\cdot)=v_{k}\left(w_{k}-e_{k}-\theta_{k}\left(x_{m}, x_{n}\right)\right), \quad u_{k, l}^{\prime}(\cdot)=\frac{d u_{k}\left(w_{k}-e_{k}-\theta_{k}\left(x_{m}, x_{n}\right)\right)}{d x_{l}}, \quad$ and $\quad v_{k, l}^{\prime}(\cdot)=$ $\frac{d v_{k}\left(w_{k}-e_{k}-\theta_{k}\left(x_{m}, x_{n}\right)\right)}{d x_{l}}$, where $k, l \in(m, n)$.

Differentiate (10) with respect to $x_{m}$ :

$\theta_{n, m}^{\prime}\left(x_{m}, x_{n}\right)=\frac{p_{m, m}^{\prime}\left(u_{m}(\cdot)-v_{m}(\cdot)\right)-\theta_{m, m}^{\prime}\left(p_{m} u_{m, m}^{\prime}(\cdot)+\left(1-p_{m}\right) v_{m, m}^{\prime}(\cdot)\right)}{p_{n} u_{n, m}^{\prime}(\cdot)+\left(1-p_{n}\right) v_{n, m}^{\prime}(\cdot)}$

Then for $n$ be willing to pay for $m$ 's risk reduction $\left(\theta_{n, m}^{\prime}\left(x_{m}, x_{n}\right)>0\right)$, it is required that $\frac{p_{m, m}^{\prime}\left(u_{m}(\cdot)-v_{m}(\cdot)\right)}{p_{m} u_{m, m}^{\prime}(\cdot)+\left(1-p_{m}\right) v_{m, m}^{\prime}(\cdot)}>\theta_{m, m}^{\prime}$. That is, there is an upper bound for the marginal WTP of $n$, motivated by a significant difference in the utilities rendered in both states of nature, the size of the marginal increase of his own non-infection probability and inversely related to the expected marginal utility. This implies $m$ should be willing to pay some positive amount, greater than zero for $x_{m}$ to occur; if not, then $\theta_{n, m}^{\prime}\left(x_{m}, x_{n}\right)<0$, implying that $n$ is not willing to pay for $x_{m}$ either. That is, $m$ should send some clear and observable signal to $n$, for this latter to show some altruistic attitude in terms of procuring $m$ some risk reduction.

Next, in order to describe the behavior of the marginal WTP with respect to altruism, set these conditions: $\quad \theta_{k, l l}^{\prime \prime}\left(x_{m}, x_{n}\right)=\frac{d^{2} \theta_{k}\left(x_{m}, x_{n}\right)}{d x_{l}^{2}}, p_{k, l}^{\prime \prime}=\frac{d^{2} p_{k}\left(e_{k}, x_{k}, \bar{p}_{k}\right)}{d x_{k}^{2}}, u_{k, l l}^{\prime \prime}(\cdot)=$ $\frac{d^{2} u_{k}\left(w_{k}-e_{k}-\theta_{k}\left(x_{m}, x_{n}\right)\right)}{d x_{l}^{2}}, v_{k, l l}^{\prime \prime}(\cdot)=\frac{d^{2} v_{k}\left(w_{k}-e_{k}-\theta_{k}\left(x_{m}, x_{n}\right)\right)}{d x_{l}^{2}}$, where $k, l \in(m, n)$. 
Differentiate (11) with respect to $x_{m}$ :

$$
\begin{aligned}
\theta_{n, m m}^{\prime \prime}\left(x_{m}, x_{n}\right) & =\frac{1}{\left(p_{n} u_{n, m m}^{\prime}+\left(1-p_{n}\right) v_{n, m m}^{\prime}\right)^{2}} \cdot \\
& \cdot\left(\left(p_{m, m m}^{\prime \prime}\left(u_{m}-v_{m}\right)-2 p_{m, m}^{\prime} \theta_{m, m}^{\prime}\left(u_{m, m}^{\prime}-v_{m, m}^{\prime}\right)-\theta_{m, m m}^{\prime \prime}\left(p_{m} u_{m, m}^{\prime}+\right.\right.\right. \\
& \left.\left.\left(1-p_{m}\right) v_{m, m}^{\prime}\right)-\theta_{m, m}^{\prime}\left(p_{m} u_{m, m m}^{\prime \prime}+\left(1-p_{m}\right) v_{m, m m}^{\prime \prime}\right)\right) \cdot \\
& \cdot\left(p_{n}^{\prime}\left(u_{n, m}^{\prime}-v_{n, m}^{\prime}\right)-\theta_{n, m m}^{\prime}\left(p_{n} u_{n, m m}^{\prime \prime}+\left(1-p_{n}\right) v_{n, m m}^{\prime \prime}\right)\right)
\end{aligned}
$$

The signs of the numerators of the first and third term on the right hand side of (12) are both positive, but it is not clear for the second one. This ambiguity is resolved if we assume $u_{m, m}^{\prime} \approx v_{m, m}^{\prime} \approx 0$, that is, the size of $x_{m}$ is not significant enough in order to induce an important increase of $p_{m}$ and, consequently, some significant WTP for the intervention that generates $x_{m}$. In this case, (12) reduces to

$$
\begin{aligned}
& \theta_{n, m m}^{\prime \prime}\left(x_{m}, x_{n}\right)=\frac{1}{\left(p_{n} u_{n, m m}^{\prime}+\left(1-p_{n}\right) v_{n, m m}^{\prime}\right)^{2}} \cdot\left(p_{m, m m}^{\prime \prime}\left(u_{m}-v_{m}\right)\right) \\
& \cdot\left(p_{n}^{\prime}\left(u_{n, m}^{\prime}-v_{n, m}^{\prime}\right)-\theta_{n, m m}^{\prime}\left(p_{n} u_{n, m m}^{\prime \prime}+\left(1-p_{n}\right) v_{n, m m}^{\prime \prime}\right)\right)<0
\end{aligned}
$$

Now, as $x_{m}$ increases significantly, $p_{m}$ also increases up to a level where $p_{m, m}^{\prime}$, $\theta_{n, m}^{\prime}\left(x_{m}, x_{n}\right)$ and $\theta_{n, m m}^{\prime \prime}\left(x_{m}, x_{n}\right)$ will approach zero, implying the policy has reached its maximum effectiveness, where it does not necessarily imply that $p_{m}=1$. However, during this process, for intermediate values of $x_{m}$ the sign of $\theta_{n, m m}^{\prime \prime}\left(x_{m}, x_{n}\right)$ is ambiguous, because $n$ may also direct his budget to $x_{n}$.

\section{CONCLUSIONS AND DISCUSSION}

A key feature of any policy to intervene in controlling diseases such as HIV/AIDS relates to the certainty of its outcomes. The essential point is whether outcomes are observable and quantifiable (i.e. there are known ex post risk-reduction or improvements in the quality of life of patients), especially when an intervention policy's design, implementation, and evaluation may be confounded with the issues of interpretability, their connection to behavior modification, and public agency implementation issues (e.g. Dolcini et al., 2010).

Our WTP model results conform with Tremblay and Ling (2005) in the sense that for any individual to evaluate risk decisions, she must observe or perceive a significant difference of utility levels between being HIV infected or not, which then imply the use of preventative measures. One implication is that the latter result is more pronounced for those individuals who are initially facing a high infection risk; however, the risk changes generated by policies have marginally decreasing effects on the probability of remaining healthy (Prohaska 1990).

As policy issues, whether the perceived effectiveness is measurable, the policy maker depends on specifics of each policy and the acceptability and reactions created when implemented in a particular context. That is, though a particular policy is intended to decrease infection risk, the population responses may differ from what is theoretically expected 
(Mawji et al 2012). For instance, in some cases IEC policies fail because the choice between risky and non-risky sexual behavior involves an instantaneous decision that takes into account only the immediate satisfaction and not the long-term effects around sexual and behavioral decisions (Fernandez 2012, Levy 2002). Finally, whether individuals correctly perceive key differences related to HIV implementation policies are likely country and region-specific (e.g. Dunn and Tan, 2010).

Altruism may play an important role in the determination of average WTP for a larger population, i.e. not just that WTP calculated for victims and potential victims of the disease (Araña and Leon 2002). However, a substantial contribution to WTP only arises when the beneficiary of the policy (i.e. those with low $p_{k}$ ) also incurs in some WTP. This would reflect an interest into decreasing the own-infection risk and, if observable, would motivate others to be willing to pay for this particular policy. In any case, apart from epidemiological considerations (Jamison 2006) for resource allocation across policies, quantification of marginal risk changes generated by the (set of) policies should be made available for health officials and policy makers.

Some important limitations of the model for this paper are as follow. First, the model is static: it does not capture dynamics related to health protection. Expanding the model into a more dynamic framework would allow comparison of intervention programs that accomplish goals with quite different time profiles. Second, no empirical evidence is provided here. We encourage others to pursue this, as we shall. Third, other factors not explicitly discussed above may also affect WTP, such as the availability of public health insurance or coverage, and how this relates to the relationship between infected patients and government agencies that might provide a suite of health care options. Moral hazard issues might arise in systems that had assured (public or government) health insurance or health care coverage. We note also that Figure 1 does not consider individual reactions in the case that intervention policies are not perceived as effective, despite the risk change. These could be observable, but in the event they are not, we recommend the use of empirical models that allow for unobservable heterogeneity. In summary, there are a host of further extensions that could be pursued in another paper.

\section{Acknowledgements}

The authors thank James Hammitt, Paul Jakus, Jason Shogren, Paan Jindapon and Kerry Smith for helpful comments on a much earlier version of the manuscript.

\section{Conflicts of Interest}

Authors do not have any conflict of interest

\section{References}

Ainsworth M, Vaillancourt D A, Gaubatz J H. (2005) "Committing to results: improving the effectiveness of HIV/AIDS assistance: an OED evaluation of the World Bank's assistance for HIV/AIDS" The World Bank Publications.

Araña J E, León C J. (2002) "Willingness to pay for health risk reduction in the context of altruism" Health Economics 11(7): 623-635. 
Bignami-van Assche S, Chao L W, Anglewicz P, Chilongozi D, Bula A. (2007) "The validity of self-reported likelihood of HIV infection among the general population in rural Malawi" Sexually Transmitted Infection 83(1): 35-40.

Corso P S, Hammit J K, Graham J D, Dicker R C, Goldie S J. (2002) “Assessing preferences for prevention versus treatment using willingness to pay" Medical Decision Making 22(5): 92-101.

De Vogli R, Birbeck G L. (2005) "Potential Impact of Adjustment Policies on Vulnerability of Women and Children to HIV/AIDS in Sub-Saharan Africa" Journal of Health, Population and Nutrition 23(2): 105-120.

Dolcini M, Gandelman A A, Vogan S A, Kong C, Leak T N, King AJ, Desantis L, O'Leary A. (2010) "Translating HIV interventions into practice: Community-based organizations' experiences with the diffusion of effective behavioral interventions (DEBIs)" Social Science and Medicine 71(10): 1839-46.

Dunn R A, Tan A. (2010) "Cervical cancer screenings in Malaysia: Are Targeted Interventions Necessary?" Social Scence and Medicine 71(6): 1089-93.

Fernandez, Mario (2012) "Relación entre el Conocimiento de los Mecanismos de Transmisión del VIH y la Percepción del Riesgo de Infección en Guayaquil, Ecuador" Revista Chilena de Salud Publica 16(2): 107-114.

Graham, D. A. (1981) "Cost-benefit analysis under uncertainty" The American Economic Review, 71(4), 715-725.

Hammitt J K, Treich N. (2007) "Statistical vs. identified lives in benefit-cost analysis" Journal of Risk and Uncertainty 35(1): 45-66.

Hurley J, Mentzaki E. (2011) "Existence and Magnitude of Health-related Externalities: Evidence from a Choice Experiment" Working Paper Series 2011-01, Department of Economics and Centre for Health Economics and Policy Analysis, McMaster University.

Jamison D T, Bremam J G, Measham A R, Alleyne G, Claeson M, Evans D B, Jha P, MIlls A, Musgrove P (eds.). (2006) "Disease Control Priorities in Developing Countries" The World Bank Group.

Johnston K M, Lima V D, Hogg R S, Tyndall M W, Gustafson P, Briggs A, Montaner J. 2010. Expanding access to HAART: a cost-effective approach for treating and preventing HIV. AIDS 24(12):1929-1935.

Jones-Lee M W. (1992) "Paternalistic Altruism and the Value of Statistical Life" The Economic Journal 102(410): 80-90.

Levy A. (2002) "A lifetime portfolio of risky and risk-free sexual behaviour and the prevalence of AIDS" Journal of Health Economics 21(6): 993-1007

Mawji E, McKinnon L, Wachihi C, Chge D, Thottingal P, Kariri A, Plummer F, Jaoko W, Ngugi E, Kimani J, Gelmon L, Nagelkerke N, Kaul R. (2012) "Does antiretroviral therapy initiation increase sexual risk taking in Kenyan female sex workers? A retrospective casecontrol study" BMJ Open 2 doi:10.1136/bmjopen-2011-000565 
McCabe C J, Goldie S J, Fisman D N. 2010. The Cost-Effectiveness of Directly Observed Highly-Active Antiretroviral Therapy in the Third Trimester in HIV-Infected Pregnant Women. Plos One 5: e10154.

Pratt, J. W., \& Zeckhauser, R. J. (1996) "Willingness to Pay and the Distribution of Risk and Wealth" Journal of Political Economy 104(4): 747-763.

Prinja S, Bahuguna P, Rudra S, Gupta I, Kaur M, Mehendale S M, Chatterjee S, Panda S, Kumar R. (2011) "Cost effectiveness of targeted HIV prevention for female sex workers in India” Sexually Transmitted Infections 87(4): 354-361.

Prohaska T R, Albrecht G, Levy J A, Sugrue N, Kim J H. (1990) "Determinants of SelfPerceived Risk for AIDS" Journal of Health and Social Behavior 31(4); 384-394.

Shiell A, Rush B. (2003) "Can willingess to pay capture the value of altruism? An exploration of Sen's notion of commitment" The Journal of Socio-Economics 32(6): 647660.

Tremblay C H, Ling D . (2005) "AIDS education, condom demand, and the sexual activity of American youth" Health Economics 14(8): 851-867.

UNAIDS. (2012) World Aids Day Report: Results. www.unaids.org 